ON THE USE

or

\title{
THE SULPHATE OF COPPER
}

\author{
ม* \\ CHRONIC DIARRHOEA.
}

B)

JOHN Elliotson, M. D. Cantab.

FELLOW OF THE ROYAL COLLEGE OF PHYSICIAN, AND PHYSICIAN to

ST. THOMAS's HOSPYTAL.

Read April 10, 1827.

ON Thursday, October 13th, 1824, I admitted into St. Thomas's Hospital, an Irishman, named Garret Welsh, twenty-four years of age, who had been labouring under diarrhœa for three months.

The abdomen was tender on pressure, the pulse quick, the skin hot, and the tongue foul and dry. The motions were yellowish and watery. I ordered a very large blister to be applied over the abdomen, half a grain of Opium to be taken night and morning, with chalk mixture, and the diet to consist of milk. 
In a few days the tenderness was much diminished, but the diarrhœa continued. Powdered Catechu was added to the mixture, and taken every six hours, and the blister was repeated, but without good effect. Infusion of galls was now made the vehicle of the Catechu, and instead of the solid Opium, taken latterly in the dose of one grain, night and morning, $f \ni j$ of the tincture was given in such astringent draught, a third blister was applied, and as considerable debility and emaciation had taken place, animal diet was substituted for the milk.

The 30th of November had now arrived, and though there was no pain or tenderness, the diarrhoea was as profuse as ever, and the man appeared likely to sink. Mr. Henry South, one of the pupils, mentioned to me that two cases of the successful exhibition of Sulphate of Copper and Opium in diarrhœea, had recently been related in the Physical Society of Guy's Hospital, and I instantly resolved to profit by his information, and prescribed half a grain of the Sulphate of Copper twice a-day, with two grains of Opium, so that the quantity of the latter might be much the same as what had previously been taken in the twenty-four hours. In a few days the disease was evidently less severe, and in eleven from the commencement of the use of the Copper, was so much abated, and the man's appearance and strength so much improved, that $I$ ventured to diminish the Opium to one grain, in- 
creasing, however, the dose of the Copper to one grain, lest the disease should gain ground again, and that I might fully satisfy myself which of the remedies had the greater share in producing the benefit. In a week more the disease had so diminished that I ventured to omit the Opium; and in another the man felt so strong, and found his disease so inconsiderable, that he would stay no longer in the Hospital, and was made an outpatient.

This case made a great impression upon me, because two powerful astringents in common use, combined with Opium, had previously failed, and the benefit clearly arose from the Sulphate of Copper, and not from the Opium, which I exhibited with it solely for the purpose of preventing the acrid quality of the salt from counteracting its astringent effects upon the intestines.

On December 8th, 1825, I admitted a sailor, named Peter Hurly, aged 48, whohad been affected many weeks with the form of diarrhœa so accurately described by Dr. Baillie, in the fifth volume of the Transactions of the College of Physicians. The description is preceded by the remark, that it " is not very generally known, and is almost constantly fatal." Dr. Baillie states, that it occurs more frequently in men than in women, and generally in persons who have long resided in hot climates; that the stools are very copious and numerous, 
pale, like a mixture of water and lime, frothy, and often of a sour smell; but if they acquire the consistence of pudding, they are still pale; and if they become figured and dark, the colour is rarely so deep as in health, and they soon again become white and frothy; that the body is thin, and the countenance sallow; that there is no tumefaction or pain of the abdomen; and that though the disease may continue several years, and be occasionally less severe, it almost constantly in the end exhausts the constitution.

The present patient was a man, and had lived much in hot climates; was thin and sallow, and had . gradually lost his flesh and strength; his motions very frequent and copious; liquid, white and frothy: he had no swelling of the abdomen, nor tormina, nor pain upon pressure.

I ordered half a grain of Sulphate of Copper, with one grain of Opium, to be taken twice a-day; and his diet to consist of milk, arrow root, strong beef-tea, and a little wine. In four days the dose was increased to one grain. He had not taken this quantity above five days before the stools were reduced to two or three in the twenty-four hours, and they were never afterwards more numerous, except for one day, probably from some accidental circumstance. In a few more days they became thicker, and from that time there was generally but one in the twenty-four hours. On the $3 d$ of 
January, rather less than four weeks from his ad. mission, they assumed a deep healthy yellow-an occurrence rarely, if ever, witnessed by Dr. Baillie,and they continued of this colour. They however became liquid again, and I augmented the dose to one grain and a half; and this not rendering them consistent, I augmented the dose after eleven days to two grains. Some pain was now felt in the epigastrium, and a blister was applied without benefit; and as it appeared to arise from the acrimony of the salt, the dose of Opium was made gr. iss, with the effect of removing it entirely. The man found himself so strong that he desired to be discharged on the $2 \mathrm{~d}$ of February. He had gained flesh, and now passed but one motion in the twentyfour hours, solid, and of a healthy appearance. He took with him a supply of medicines for a fortnight.

This case is particularly satisfactory when compared with Dr. Baillie's experience. "The influence of medicine," he says, " is generally very inconsiderable. Patients will sometimes receive advantage from very small doses of mercury, which will sometimes stimulate their liver to a better and more plentiful secretion of bile, without impairing the strength of their constitution. Some advantage too is occasionally derived from the different kinds of bitter medicines, as cascarilla, cusparia, \&c. combined with a few drops of laudanum. A medicine 
of this kind, taken twice a-day, will sometimes im. prove the digestion, when it is deficient, will render the motions more solid and less frequent, and increase the strength of the constitution: but this good effect is hardly ever permanent, for the patients almost constantly fall back into the state of frequent and frothy stools. The repeated returns of the complaint at length wear out the constitution, and the patients sink."

The stools of this man became highly bilious, without the exhibition of a grain of mercury, and acquired not only a biliousness, but a consistence never, I should imagine, witnessed by Dr. Baillie; and this permanently. His strength and flesh were restored, and not by general tonics, but by the removal of the disease.

Although the Opium must have been useful, the great benefit cannot be ascribed to it, because Dr. Baillie gave Opium with such results as we have seen, and because the stools did not become consistent till the dose of the Copper was increased.

The following case is one of dysenteric diarrhœa.

John Roberts, aged 39, was admitted April 14th, 1826. - He had been frequently in North America, but in England ever since October. He had laboured for three months under severe purging, the 
stools being bloody, and amounting to about twelve in the day and night, with tormina and tenesmus. At present the stools were generally thin and yel. low, though often bloody and slimy.

I ordered him one grain of Sulphate of Copper, and one grain of Opium, twice a-day. The day after his admission the stools were reduced to five. In three days more they had increased to seven or eight. The medicines were therefore given three times a-day; and there being no amendment at the end of a week, the dose of the Copper was increased to one grain and a half; in another week to two grains; and in three days more (now three weeks since his admission), to two grains and a half, as the stools varied from six to nine in the twenty-four hours, and were frequently liquid; though a sufficient quantity of solid healthy fæces was daily discharged; he had experienced no tormina nor tenesmus since his admission, had grown strong, and gained flesh. In ten days the dose was raised to three grains, the Opium remaining all along at the original quantity. In a week from this period the stools were reduced to three, and in a few days more one only was passed in the twenty-four hours.

He left the Hospital June 1st, with a supply of medicine, perfectly well and grown fat, though he had taken only the house allowance of meat every other day, and table beer. 
Notwithstanding his stools had been generally bloody and slimy for three months previous to his admission, they were never so during his stay, nor did he ever experience the tenesmus, and but once the tormina.

The following was a case of long and severe diarrhœa.

A Swede, named John Nelson, aged thirty-six, was admitted January 19, 1825. He had been left by his ship, with eight others, on a desolate island in the Indian Ocean, where he remained four years and three months; then went to the Isle of France, and was attacked with dysentery, which ceased at the Cape, and returned at Amsterdam. He had now suffered from diarrhœa six months. The motions amounted to twelve in the day and night, were yellow, and attended with pain and scalding. .

The Sulphate of Copper was prescribed in the quantity of one grain, with one grain of opium twice a-day. The dose was augmented to one grain and a half, and subsequently to two grains; and at length one grain and a half, with but half a grain of opium, was given three times aday. From this time the motions were less copious, and their number in a few days was reduced to two or three in the twenty-four hours, and on March 14, he was considered perfectly well, 
though he remained some time longer in the house on account of a diseased tibia.

The next case illustrates the use of the remedy after the failure of others.

A man, named Henry Hurle, aged thirty-seven, was admitted October 6,1825 , on account of frequent bloody and mucous stools, under which he had suffered for five weeks. He had been in the Brazils, the West Indies, North America, and the Mediterranean, but had resided in England for nine years, and worked last at the iron works in Wales.

He was ordered one scruple of hydrargyrum cum creta and one grain of opium twice a-day. The quantity of opium was increased to one grain, to one grain and a half, and finally to two grains; and a half a pint of dec. hæmatoxyli was drunk daily.

On the 15th of November he had not improved, and I prescribed half a grain of Sulphate of Copper, with but one of opium. The dose of the salt was soon increased to one grain, and that of the opium to one grain and a half.

He was discharged perfectly well on December 22. 
Another man, named Aaron Newman, aged thirty-five, who had been in the West Indies, and attacked with dysentery there, was admitted March 23, 1826, for profuse diarrhœa of eight months' standing.

He took one grain of the Sulphate of Copper, twice a-day, with one grain of opium; then one grain and a half; and then this quantity, with the original dose of opium, three times a-day; and left the hospital quite well April 27.

The last with which I will trouble the Society, was one of four years' standing, but at intervals. The man, named John Mundy, was thirty years of age, and the stools were bloody, and not fewer than ten every day.

I prescribed one grain of the Sulphate of Copper, with one grain of opium, twice a-day.

In five days, as he was no better, he took his medicines three times a-day, and in four more, the dose of the salt was increased to one grain and a half. In a fortnight the stools were reduced to two or three in the day and night, and they never afterwards exceeded this number; they also ceased to be bloody, though formerly they were invariably so. The dose of the salt was augmented to two grains and a half; the motions acquired consist- 
ence and their natural frequency; the man felt well and strong, and was discharged on the 18th of May.

I could greatly extend this list, but the narration of cases is always tedious, especially when they are so similar, as such must necessarily be to each other; and the preceding sufficiently illustrate the power of the remedy. I will therefore content my. self with mentioning the general results of my experience during the last two years and a half in St. Thomas's Hospital, where a great number of sailors are admitted labouring under intestinal affections consequent upon dysentery in hot climates.

I am satisfied that the Sulphate of Copper is superior to every other astringent in chronic diarrhœa,- - that it will cure the disease more quickly than any other, and often when all others fail.

Three grains three times a-day is the largest quantity I have prescribed; but this I have frequently given. The dose usually borne and,required varies from a grain and a half to three grains. It may be taken for an indefinite time without any fear of constitutional ill effects.

I had twovery severe cases, in which the quantity. of blood and matter in the evacuations, and the wretched appearance of the countenance rendesed 
the existence of great disease of the inner surface of the intestines probable, and which would most likely have proved fatal but for the remedy. It arrested the complaint after a time, but such had been the severity of the disease, and so much reason was there for apprehending mesenteric affection, and thickening of all the substance of the intestines, that, if it was omitted for a week, the diarrhœa in some measure returned. These patients took it between three and four months, in doses of two and three grains three times a-day, with no other effect than that of controlling the disease, and improving the appearance in a degree which surprised every one *.

It certainly has a tendency to produce vomiting and griping. On these accounts I have always combined it with opium, and never, but in the first case, and then only for a week and at the cessation of the disease, ventured to exhibit it alone $t$. No inconvenience resulted, and I have generally found

* July 21,1827. I take advantage of the interval which has elapsed before the paper is printed, to add that these men have both recovered, after taking the medicine about six months. Without it, I am convinced that they would gradually have sunk.

+ With the first of the patients mentioned in the preceding note, I gradually lessened the quantity of opium till it was omitted altogether, and he took three grains of the sulphate alone three times a-day, for the last six weeks that he was in the hospital. 
a grain and a half of opium sufficient to prevent even three grains from griping in the least. Notwithstanding the unquestionable cooperation of the opium with the sulphate, the far superior share of the salt in curing the disease has been repeatedly proved by the previous failure of opium alone, or combined with other astringents, and by the dose of opium being actually diminished when the copper was superadded.

The disposition to occasion sickness is much diminished by administering it, like all medicines inclined to disagree with the stomach, and intended to pass the organ without sensible effect, in the form of pill and after food has been taken. A dose of it, as well as of oxymuriate of mercury or tartrite of antimony, which would cause sickness, if taken in a liquid form or before breakfast, will be borne perfectly well, and frequently indeed a much larger dose, if taken in the form of pill and after a meal.

In thus recommending a medicine for a purpose which, as far as I know, has not been mentioned by any author, I am anxious, as upon every such occasion, however great its excellence, not to excite too high an expectation,-not to appear pretending that it is universally applicable, and that it never fails. In cases only in which astringents are proper is it proper; and although ulceration of the inner coat of the intestines, and very extensive 
ulceration, will heal *, and the Sulphate of Copper will contribute to the cure more than any other medicine, the mischief may be irremediable by any measures ; and, on the other hand, chronic diarrhœea is frequently kept up by the state of mind, by the mode of living, or by the residence. No medicine can alter these, and I knew two examples of the failure of it and all other remedies, when a removal from town cured the disease in a few days, and a return to the metropolis was invariably followed by a return of the diarrhœa.

Grafton Street, April 10, 1827.

P. S.-IN the paper upon the Subcarbonate of Iron that the Society did me the honour of printing, I made an additional and confirmatory report upon prussic acid and pulvis antimonialis. Perhaps I may be at present allowed to do the same with respect to quinina and the subcarbonate of iron.

Since the appearance of the paper upon quinina, $I$ have attended near a hundred and fifty cases of

* Instances may be found in the writings of Dr. Andral, in Dr. Peter Latham's work upon the disease of the Penitentiary, and in the Journal Général de Médecine of last year. The most extensive, I believe, is related in Mr. Huwship's book on Morbid Anatomy :-a very long tract of ulceration was found healed. 
ague, and treated all with the sulphate. Many were combined with so much inflammation in the abdomen, chest, or head, as to require venæsection, some with dropsy, some with a chronic disease of the lungs or liver, but every one was cured. Having never seen it augment any inflammation that might be present, or interfere with antiphlogistic measures, I have always given it under all circumstances, and simultaneously adopted any other measures that might be demanded by other symptoms. Some cases, and those were generally quartans, would not yield to less than five grains every four hours; but this quantity has never failed after being exhibited a week or ten days. Like mercury in syphilis, I have frequently seen its use followed by a relapse, when not continued a proper length of time after the cessation of the disease. It cannot be a matter of surprise that a fresh exposure to the causes will also renew ague after a cure, precisely as happens with syphilis after the successful exhibition of mercury. The disease in London may in general be arrested immediately, by the exhibition of ten grains at once, just before or after the paroxysm.

Dr. Home* found the bark much more successful after than before the paroxysm, and this is my experience with quinina. I am convinced that the best practice is first to give ten grains as soon as the paroxysm is over. This almost always (the ex-

- Home's Clinical Experiments, Histories, and Dissections. 
ceptions are generally quartans) prevents the paroxysm next expected, and if repeated daily at the same hour, often cures the disease. But as ten grains given in any number of doses in the twenty-four hours are frequently insufficient to conquer it, and half a drachm may be required, it is sometimes necessary, in addition to these ten grains after the fit, to make the whole quantity in the twenty-four hours amount to a scruple or half a drachm, by small doses also, every six or eight hours.

The chief object of the paper upon the subcar-. bonate of iron, was to show that it might generally be given in doses of three or four drachms, and that it was almost a specific for chorea. The former point is now considered to be established, and $I$ have since attended nine cases of the disease, every one of which yielded to the remedy. Some, like the former cases, were attended by head-ache, some had resisted other means. The time usually required varied from six to ten weeks : one was cured in four, and one in not fewer than twelve. I could not then satisfy myself that large doses were superior to small ones in any disease, but I have now met with several instances of disease yielding at once to large doses, after resisting those in common use. Its importance, to which also I then alluded, in many cases of chronic ulceration, has been again repeatedly proved. I have treated a genuine case of traumatic tetanus with it, 
in doses of half an ounce every two hours, taken diffused with treacle in beef-tea, and the mitigation of the disease upon its exhibition was too obvious for me not to ascribe the cure to the remedy. I have always conceived that we are upon a wrong scent in our attempts to cure tetanus and hydrophobia by narcotics, and that we should employ other remedies which exert peculiar actions upon the nervous system. The full powers of iron $\mathrm{de}_{\mathbf{z}}$ serve to be put fairly to the test, when its exhibition is practicable.

There is a remedy upon which $I$ am anxious to make a report, because I have had ample experience of its utility. During the last three years I have employed Acupuncture very extensively, both in private and at St. Thomas's Hospital, in rheumatism. My experience perfectly coincides with that of Mr. Churchill, - of it being chiefly useful in the rheumatism of fleshy parts-rheumatalgia; and the more so, as the disease is less inflammatory. Indeed when the parts are hot, or the pain is increased by heat, the remedy is generally useless, and cannot supply the place of antiphlogistic measures. On the same principle I have never seen it beneficial in any inflammation or inflammatory pain. Like Mr. Churchill, I find that one needle allowed to remain an hour or two in a part, is more efficient than several used but for a few minutes. The effects are often magical. The pain sometimes ceases while the needle 
is in the flesh, but generally three or four applications to each painful part are required. I have known the disease not entirely yield before the ninth. Of forty-two cases taken in succession from the Hospital books, thirty were cured; and the other twelve were clearly not adapted for it, as they were either accompanied or aggravated by heat, and yielded afterwards to antiphlogistic measures. It is accasionally a good mode of letting off the fluid of anasarca : but for this purpose the needle requires to be passed merely through the skin, and not to an inch or an inch and a half, as in rheumatism. Neither is its use always attended with success, as in rheumatism. When practised below the knees, I have heard of it producing mortification; but above them, and in the arms and trunk, it appears free from danger. 